\title{
InP/InGaAlAs distributed Bragg reflectors grown by low-pressure metal organic chemical vapor deposition
}

\author{
T.C. Lu*, J.Y. Tsai, J.T. Chu, Y.S. Chang, S.C. Wang \\ Institute of Electro-Optical Engineering, National Chiao Tung University, 1001 Ta Hsueh Rd., Hsinchu 30050, Taiwan, ROC
}

Received 4 October 2002; accepted 4 December 2002

Communicated by D.W. Shaw

\begin{abstract}
Long-wavelength vertical cavity surface emitting lasers (VCSELs) are considered the best candidate for the future low-cost reliable light sources in fiber communications. However, the absence of high refractive index contrast in InPlattice-matched materials impeded the development of 1.3-1.5 $\mu \mathrm{m}$ VCSELs. Although wafer fusions provided the alternative approaches to integrate the InP-based gain materials with the GaAs/AlAs materials for their inherent high refractive index contrast, the monolithic InP-based lattice-matched distributed Bragg reflectors (DBRs) are still highly attractive and desirable. In this report, we demonstrate $\mathrm{InP} / \mathrm{InGaAlAs}$ DBRs with larger refractive index contrast than $\mathrm{InP} / \mathrm{InGaAsP}$ and InAlAs/InGaAlAs DBRs. The switching between InP and InGaAlAs layers and growth rate control have been done by careful growth interruption technique and accurate in situ optical monitoring in low-pressure metal organic chemical vapor deposition. A 35 pairs $1.55 \mu \mathrm{m}$ centered InP/InGaAlAs DBRs has the stopband of more than $100 \mathrm{~nm}$ and the highest reflectivity of more than $99 \%$. A VCSEL structure incorporating 35 pairs InP/InGaAlAs DBR as the bottom mirror combined with a $2 \lambda$ thick periodic gain cavity and 10 pairs $\mathrm{SiO}_{2} / \mathrm{TiO}_{2}$ top dielectric mirrors was fabricated. The VCSELs lased at $1.56 \mu \mathrm{m}$ by optical pumping at room temperature with the threshold pumping power of $30 \mathrm{~mW}$.
\end{abstract}

(C) 2002 Elsevier Science B.V. All rights reserved.

PACS: $81.05 . \mathrm{Ea} ;$ 81.15.Gh; 42.55.Px

Keywords: A3. Distributed Bragg reflectors; A3. Metalorganic chemical vapor deposition; B3. Laser diodes

\section{Introduction}

Long-wavelength $(1.3-1.5 \mu \mathrm{m})$ vertical cavity surface emitting lasers (VCSELs) are considered the best candidate for the future light sources in fiber communications. The advantages of VCSELs

*Corresponding author. Tel.: + 886-3-5712121x56320; fax: $+886-3-5716631$.

E-mail address: timtclu.eo89g@nctu.edu.tw (T.C. Lu). include single longitudinal mode output, small divergence circular emission beam profile, low power consumption and low-cost reliable productions. The absence of high refractive index contrast in InP-lattice-matched materials impeded the progress of the development of $1.3-1.5 \mu \mathrm{m}$ VCSELs in comparison to the short-wavelength $(0.78-0.98 \mu \mathrm{m})$ VCSELs. Recently, long-wavelength VCSELs have been successfully demonstrated with several different approaches. First, 
wafer fusion technique, that integrated the InPbased active layers and GaAs-based DBRs together, had been successfully realized for highperformance long-wavelength VCSELs [1,2] but the capability of mass production is still questionable. Second, the InGaNAs $1.3 \mu \mathrm{m}$ VCSELs grown on GaAs substrates have been demonstrated with excellent characteristics [3,4]. However, to extend the InGaNAs gain peak to beyond $1.5 \mu \mathrm{m}$ is rather difficult.

Monolithically grown DBRs lattice-matched to InP continued to attract interests due to the wellexisting highly efficient InGaAsP and InGaAlAs gain materials covered the wavelength window from 1.3 to $1.8 \mu \mathrm{m}$. Metamorphic GaAs/AlAs DBRs lattice-matched to InP substrate have been applied to realize the long-wavelength VCSELs $[5,6]$ but the inherent dislocations in metamorphic layers have impacts on the reliability of the devices. The Sb-based DBRs have large refractive index contrasts $\Delta n$ ranging from 0.43 to 0.44 and have been successfully applied in the VCSEL structures [7,8]. However, these DBRs have drawbacks such as the low thermal conductivity and relatively high growth complexity. The problem with the conventional InP-lattice-matched $\mathrm{InP} /$ InGaAsP and InAlAs/InGaAlAs is the small refractive index contrast $(\Delta n=0.27$ for $\mathrm{InP} /$ InGaAsP and $\Delta n=0.3$ for InAlAs/InGaAlAs) resulting in a larger number of DBR pairs required to obtain high reflectivity. In addition, using the conventional DBRs, not only the penetration depth will increase causing more absorption, but the heat dissipation is also a problem.

Recently, the DBRs based on relatively large refractive index contrast $(\Delta n=0.34)$ material combination of $\mathrm{InP} / \mathrm{InGaAlAs}$ have also been demonstrated [9-11]. This material combination not only has a larger refractive index contrast than the conventional InP/InGaAsP and InAlAs/InGaAlAs material systems, but it also has other benefits including the smaller conduction band discontinuity, which is good for n-type DBRs, and the better thermal conductivity due to the binary alloy of InP. However, the InP and InGaAlAs belong to different group-V-based materials. Problems like the As carry over, the transitional interface, and lateral uniformity will affect the quality of the epitaxial layers and the reflectivity of the DBRs. As a result, the challenge of growing this combination relies on perfect switching between InP and InGaAlAs.

The growth interruptions have been frequently used in the metal organic chemical vapor deposition (MOCVD) growth of the InGaAs/InP or InGaAs/InGaAsP quantum wells in order to obtain abrupt interface $[12,13]$, but the growth of the InP/InGaAlAs DBRs using growth interruptions has not been investigated. In this paper, we report the effect of the growth interruptions on fabrication of the InP/InGaAlAs DBRs. The lateral uniformity and the reflectivity of the DBRs are very sensitive to the stabilization time of each terminated interface. We incorporated an in situ laser reflectometry while growing DBRs with thickness more than $8 \mu \mathrm{m}$ to insure minimum fluctuation in the center wavelength of the stopband. The optically pumped $1.56 \mu \mathrm{m}$ VCSELs with 35 pairs InP/InGaAlAs DBRs achieved stimulated emission at room temperature with the threshold pumping power of $30 \mathrm{~mW}$.

\section{Experimental procedure}

All structures were grown in a vertical-type lowpressure MOCVD system with a rotating disk. The disk rotated at 900 revolutions/min to maintain the laminar gas flow. The growth pressure was 70 Torr. The growth temperature was $625^{\circ} \mathrm{C}$. V/III ratio was 150 for InP and 200 for InGaAlAs. The growth rate was $36.5 \mathrm{~nm} / \mathrm{min}$ and $35 \mathrm{~nm} / \mathrm{min}$ for InP and InGaAlAs, respectively. The alkyl sources were trimethylindium, trimethylgallium, and trimethylaluminum, and the group $\mathrm{V}$ gases were $\mathrm{AsH}_{3}$ and $\mathrm{PH}_{3}$. The carrier gas was hydrogen. The epitaxial layers were all grown on n-type $\left(\begin{array}{lll}1 & 0 & 0\end{array}\right)$ InP substrates. The growth interruption between the InP and InGaAlAs had been divided into three steps. In the first step, the gases flowing just after the growth were hydrogen and the group V source used in the previous layer but with half of the flow rate for different periods of $t_{\mathrm{p}}$ minutes. Then, the group source was switched off. The hydrogen was kept flowing for $0.02 \mathrm{~min}$ in the second step to remove the remaining group $\mathrm{V}$ source in the 
reactor. After that, the other group V source with the same flow rate used in the next layer was switched on with the hydrogen flowing for $0.01 \mathrm{~min}$ in the third step to prepare the growth environment. Five different periods $\left(t_{\mathrm{p}}=0,0.1\right.$, $0.2,0.3$ and $0.4 \mathrm{~min}$ ) of interruption were examined.

The optical microscope with the magnification of 1200 examined the as-grown wafers first to check the lateral uniformity. The spectrometer was used to determine the reflectivity of the DBRs. The reflectivity of the $\mathrm{Au}$ film was used as the reference. The crystal quality and the lattice mismatch of the DBRs were analyzed by the double crystal X-ray. The thickness of each layer and the vertically compositional profiles were investigated by the field emission scanning electron microscope (SEM) and the secondary ion mass spectrometry (SIMS).

\section{Results and discussion}

The DBRs with ten pairs InP/InGaAlAs designed for $1.55 \mu \mathrm{m}$ VCSELs were first grown and investigated. The quarter-wavelength thickness of $\mathrm{InP}$ and InGaAlAs was 122 and $110 \mathrm{~nm}$, respectively. To avoid the absorption of DBRs while the operating wavelength was $1.55 \mu \mathrm{m}$, the latticematched $\operatorname{In}_{0.53} \mathrm{Ga}_{0.39} \mathrm{Al}_{0.08}$ As was used with a band gap emission wavelength of $1.42 \mu \mathrm{m}$. Fig. 1(a) summarizes the surface conditions of 10 pairs $\mathrm{InP} / \mathrm{InGaAlAs}$ DBRs grown with different interruption times $t_{\mathrm{p}}$ under the examinations by the optical microscope with a magnification of 1200 . When there was no interruption time $\left(t_{\mathrm{p}}=0 \mathrm{~min}\right)$, pits with diameter ranging from 0.5 to $2 \mu \mathrm{m}$ appeared densely on the surface of the as-grown wafer. When $t_{\mathrm{p}}$ increased to $0.4 \mathrm{~min}$, the pits decreased to fewer than 100 per $\mathrm{cm}^{2}$. A DBR structure grown with 0.1 -min interruption time was investigated by SEM and the picture of the cross section is shown in Fig. 1(b). The arrow indicates the location where the pits occurred. Fig. 2 shows the reflectivity curves of these samples measured by the spectrometer. The maximum reflectivity of DBRs increased when the interruption time $t_{\mathrm{p}}$ became larger. However, the center wavelength and the width of the stopband remained unchanged. Fig. 3 shows the X-ray diffraction pattern of a set of samples grown with different interruption times. The value of lattice mismatch of all samples was within $200 \mathrm{ppm}$. Meanwhile, more intense satellite peaks are observed when the interruption time is longer. As far as the DBRs of the VCSELs are concerned, it is very critical to increase the reflectivity. The decrease of the reflectivity of the DBRs and the intensity of the satellite peak of the X-ray diffraction pattern with the shorter interruption time may be resulted from the scattering loss caused by the pits on the lateral interface. Nevertheless, the small differences in the width of the stopband in the reflectivity curves among five samples showed that the layer compositions in the vertical direction did not vary a lot indicating the abruptness between the interfaces was intact. The SIMS results of InP/InGaAlAs DBRs grown with $t_{\mathrm{p}}=0.2 \mathrm{~min}$ shown in Fig. 4 suggest that the phenomena of As carry-over did not appear. Consequently, the degradation of the reflectivity of $\mathrm{InP} / \mathrm{InGaAlAs}$ DBRs mainly relates to the growth interruption time. After an InP or an InGaAlAs layer has grown, the surface needs time to stabilize under the atmosphere of the previous group $\mathrm{V}$ gas. Then, the next layer with alternate group V source can continue to grow. This is the different case from growing the InAlAs/InGaAlAs or AlAs/GaAs material system. When the interruption time is shorter than $0.1 \mathrm{~min}$, the atom of the group $\mathrm{V}$ source cannot completely occupy the surface. Meanwhile the other group V source switches on and enters the vacant site. This lattice mismatch causes the formation of defects. The defects elongate upward to form the pits while the growth continues. The further investigation of these pits and defects can be done by using the high-resolution transmission electron microscope. These results suggest that to grow the $\mathrm{InP} /$ InGaAlAs DBRs with high reflectivity, one need to add interruption time $t_{\mathrm{p}}$ more than $0.2 \mathrm{~min}$ and make compromises between the total growth time, the amount of source usage and the required high reflectivity.

Next, two DBR structures with 35 pairs of $\mathrm{InP} /$ $\mathrm{In}_{0.53} \mathrm{Ga}_{0.39} \mathrm{Al}_{0.08} \mathrm{As}$ and 35 pairs of $\operatorname{In}_{0.52} \mathrm{Al}_{0.48} \mathrm{As} /$ 

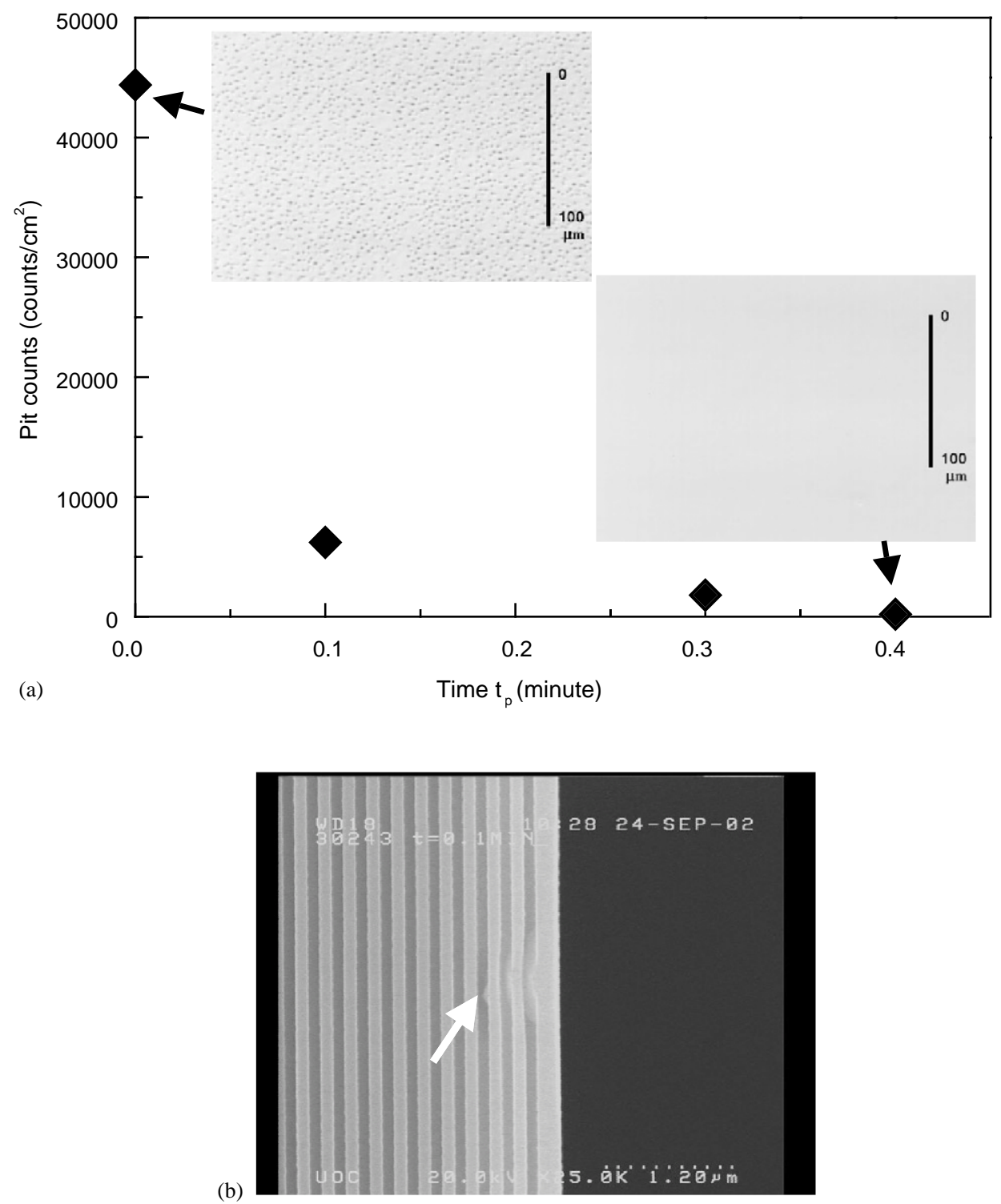

Fig. 1. (a) The pit density of 10 pairs InP/InGaAlAs DBRs grown with different interruption times $t_{\mathrm{p}}$. (b) The cross section of the InP/ InGaAlAs DBRs grown with 0.1-min interruption time investigated by SEM.

$\mathrm{In}_{0.53} \mathrm{Ga}_{0.39} \mathrm{Al}_{0.08} \mathrm{As}$ were grown for comparisons. The interruption time $t_{\mathrm{p}}$ for growing $\mathrm{InP} / \mathrm{In}_{0.53-}$ $\mathrm{Ga}_{0.39} \mathrm{Al}_{0.08} \mathrm{As}$ DBRs was chosen to be $0.4 \mathrm{~min}$. Fig. 5 shows the reflectivity curves of these two samples measured by the spectrometer. The measured reflectivity of samples was normalized to the reflectivity of the $\mathrm{Au}$ film. The maximum reflectivity of the $\mathrm{InP} / \mathrm{In}_{0.53} \mathrm{Ga}_{0.39} \mathrm{Al}_{0.08} \mathrm{As}$ DBRs can reach over $99 \%$ and the width of the stopband is more than $100 \mathrm{~nm}$, which is 1 arger than the width of the $\operatorname{In}_{0.52} \mathrm{Al}_{0.48} \mathrm{As} / \mathrm{In}_{0.53^{-}}$ $\mathrm{Ga}_{0.39} \mathrm{Al}_{0.08} \mathrm{As}$ DBRs. 


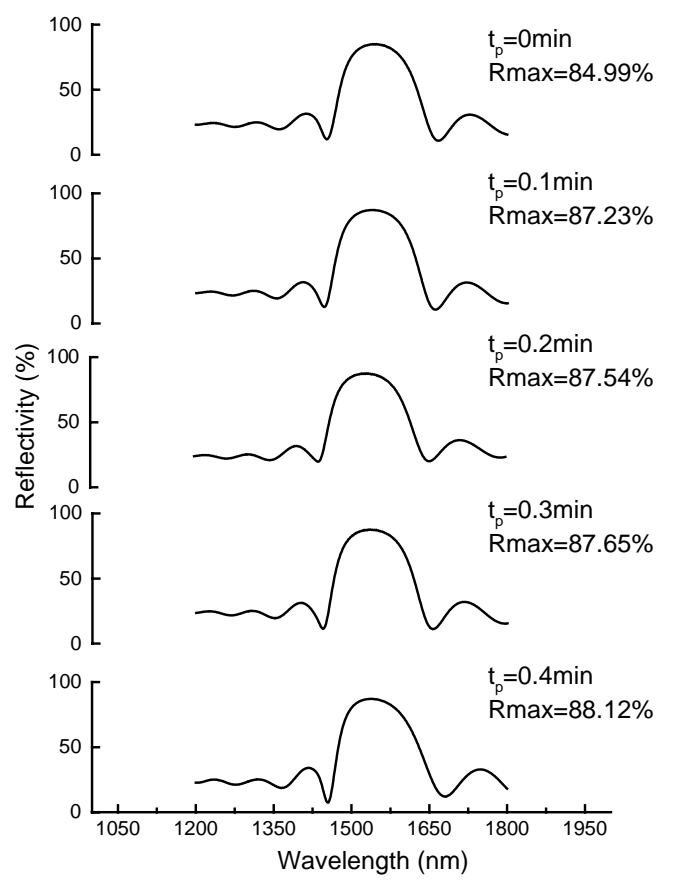

Fig. 2. The reflectivity curves of 10 pairs InP/InGaAlAs DBRs grown with different interruption times $t_{\mathrm{p}}$.

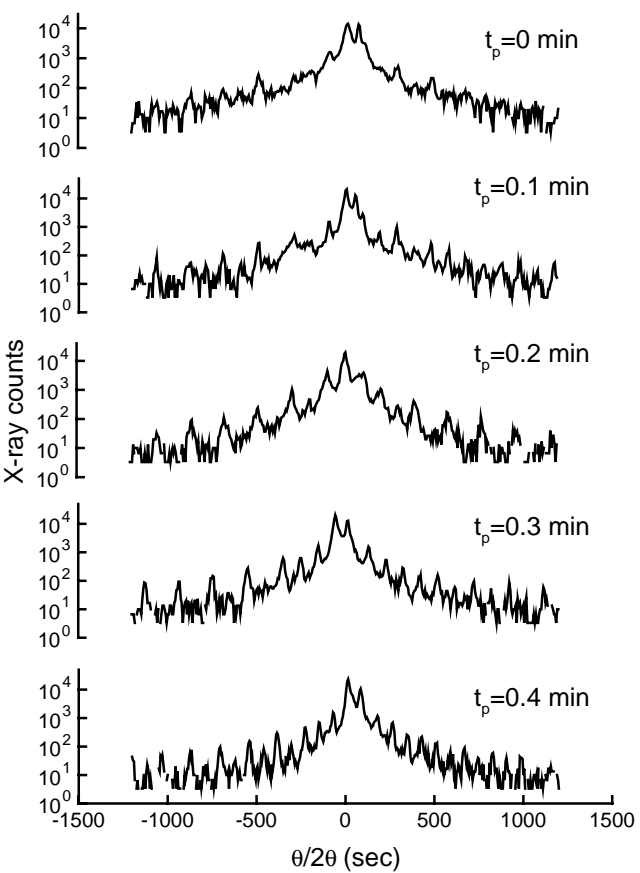

Fig. 3. The X-ray diffraction patterns of 10 pairs $\operatorname{InP} /$ InGaAlAs DBRs grown with different interruption times $t_{\mathrm{p}}$.

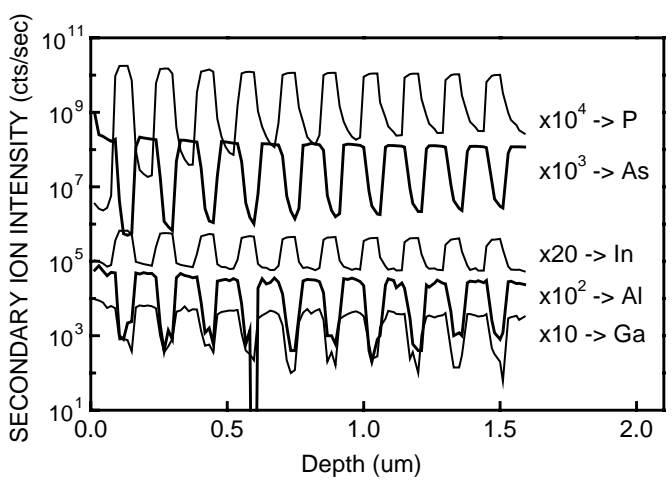

Fig. 4. The SIMS results of InP/InGaAlAs DBRs grown with $t_{\mathrm{p}}=0.2 \mathrm{~min}$.

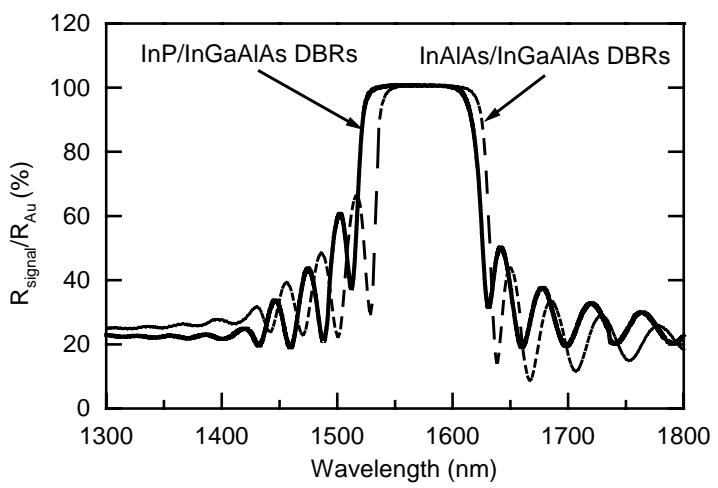

Fig. 5. The reflectivity curves of 35 pairs $\mathrm{InP} / \mathrm{InGaAlAs}$ and InAlAs/InGaAlAs DBRs measured by a spectrometer.

\section{Device performance}

To further validate the viability of the DBR structures for long-wavelength VCSELs, we have grown a laser structure based on the $\mathrm{InP} / \mathrm{InGaA}$ 1As DBRs using the growth interruption technique. Fig. 6(a) shows the laser structure. The epitaxial layers were grown on an n-type InP substrate. The first step was to grow the $5 / 4 \lambda$ thick InGaAlAs and InP calibration layers followed by the 35 pairs InGaAlAs/InP DBRs. The interruption time was $0.2 \mathrm{~min}$. The laser reflectometry in situ monitored the epitaxial growth. By collecting the reflection of a $905 \mathrm{~nm}$ laser light perpendicularly impinging on the wafer surface, one can obtain the information about the growth rate and the crystal quality of the epitaxial layers. Fig. 6(b) shows the reflectivity of $5 / 4 \lambda$ thick InGaAlAs and 
(a)
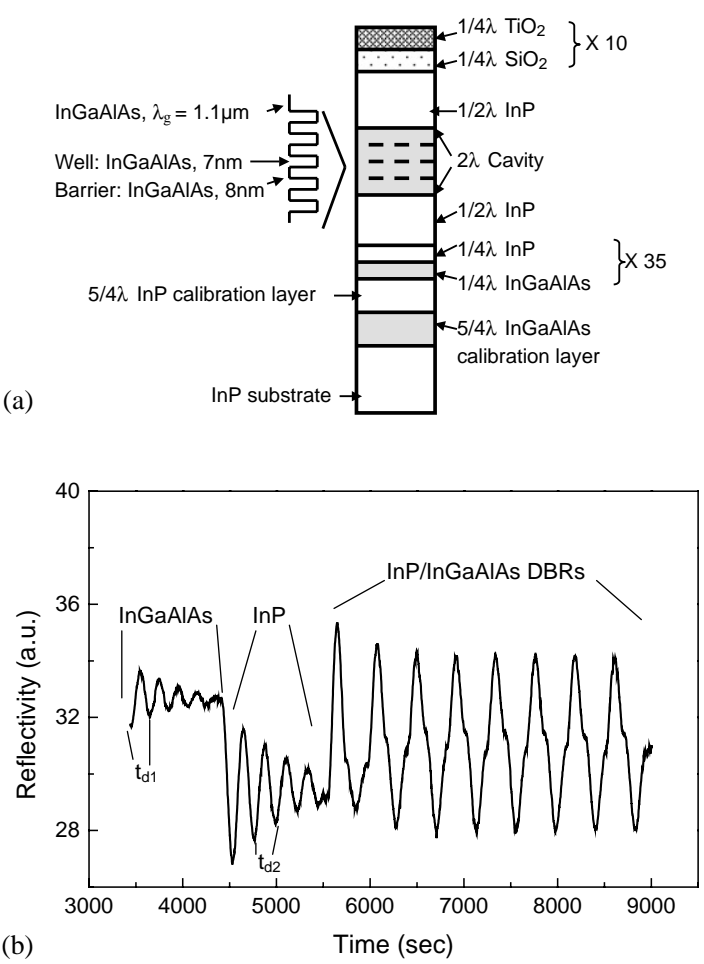

Fig. 6. (a) Schematic cross section of VCSELs. (b) In situ reflectometry versus growth time of $5 / 4 \lambda$ thick InGaAlAs and InP calibration layers followed by the DBRs.

InP calibration layers. The growth rate can be obtained by $\mathrm{GR}=\lambda T_{\mathrm{d}} / 2 N$, where $\lambda=905 \mathrm{~nm}$ and $N$ is the refractive index of the epitaxial layer at the growth temperature. The growth rate of the calibration layers and the growth parameters were used for adjusting the growth conditions of the DBRs to meet the stringent tolerance of the target emission wavelength in one epitaxial run $[14,15]$. The $2 \lambda$ thick periodic gain cavity was grown in the second step. At the same time, another InP dummy wafer was loaded in MOCVD for photoluminescence (PL) measurement. The laser structure has InGaAlAs cladding layers of $2 \lambda$ thick with a band edge emission peak at $1.1 \mu \mathrm{m}$. Three sets of strain-compensated multi-quantum wells (SCMQWs) were placed at the anti-nodes of the electric standing wave field within the $2 \lambda$ thick cavity to increase the enhancement factor of MQW active regions. Each set of SCMQWs consisted of five InGaAlAs (strain $=1.5 \%$, thick- ness $=7 \mathrm{~nm}$ ) quantum wells and four InGaAlAs (strain $=-0.6 \%$, thickness $=8 \mathrm{~nm}$ ) barriers and the PL wavelength was tuned at $1.51 \mu \mathrm{m}$, which is $40 \mathrm{~nm}$ blue-shifted from the target emission wavelength to insure the proper operation of the VCSELs at room temperature [16]. Two InP space layers of half $\lambda$ thickness grown on the top and the bottom of the cavity were served to protect the InGaAlAs layer from being oxidized during the post-processing. Finally, the wafer was coated with 10 pairs $\mathrm{SiO}_{2} / \mathrm{TiO}_{2}$ top dielectric mirrors to form a complete VCSEL structure.

Fig. 7 shows the reflectivity and PL measurements during every process stage. All of the reflectivity results were normalized to the reflectivity of Au film. Fig. 7(a) shows the reflectivity curve of 35 pairs InGaAlAs/InP DBRs. The center wavelength of the stopband is located at $1555 \mathrm{~nm}$. Fig. 7(b) shows the reflectivity of the half-cavity VCSEL. The PL spectrum of the active regions grown on the InP dummy wafer in the same run is shown in Fig. 7(c). The PL peak is $1510 \mathrm{~nm}$ and the full-width at half-maximum (FWHM) is $54 \mathrm{~nm}$. The peak of shorter wavelength was the signal belonging to the previous grown layers on the InP dummy wafer. Fig. 7(d) shows the reflectivity of the complete VCSEL structure and the Fabry-Perot dip is located at $1558 \mathrm{~nm}$. Fig. 7(e) demonstrates the PL spectrum of the complete VCSEL structure. The peak wavelength coincides with the Fabry-Perot dip and the FWHM is measured to be $3.3 \mathrm{~nm}$.

The complete VCSEL structure was placed in an optical pumping system. The pumping source was a continuous-wave-operated Ti:sapphire laser. The wavelength of the Ti:sapphire laser was tuned at $990 \mathrm{~nm}$ although the pumping wavelength at slightly beyond $1.1 \mu \mathrm{m}$ would be more desirable to avoid absorption of the $\operatorname{InP}(\lambda g=0.9 \mu \mathrm{m})$ space layers and InGaAlAs $(\lambda g=1.1 \mu \mathrm{m})$ cladding layers. The diameter of the pumping beam entered from the top dielectric DBRs was estimated to be $30 \mu \mathrm{m}$. Fig. 8 shows the pumping result of the VCSEL. The threshold pumping power is $30 \mathrm{~mW}$ at room temperature. The wavelength of the output beam is $1562 \mathrm{~nm}$. The minimum linewidth above threshold is $1 \mathrm{~nm}$ limited by the resolution of the spectrometer. The red shift of the peak 

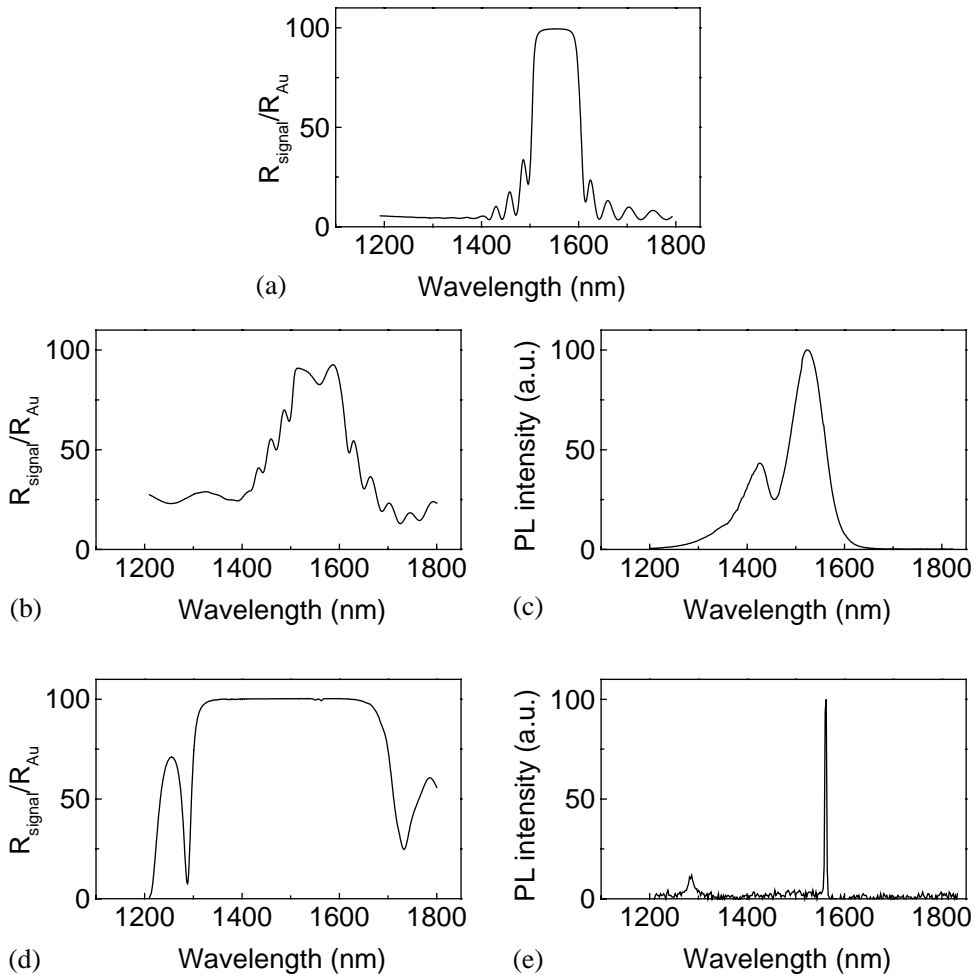

Fig. 7. (a) Reflectance spectrum of 35 pairs InGaAlAs/InP DBRs. (b) Reflectance spectrum of a half VCSEL structure. (c) PL spectrum of SCMQWs. (d) Reflectance spectrum of a complete VCSEL structure. (e) PL spectrum of a complete VCSEL structure.

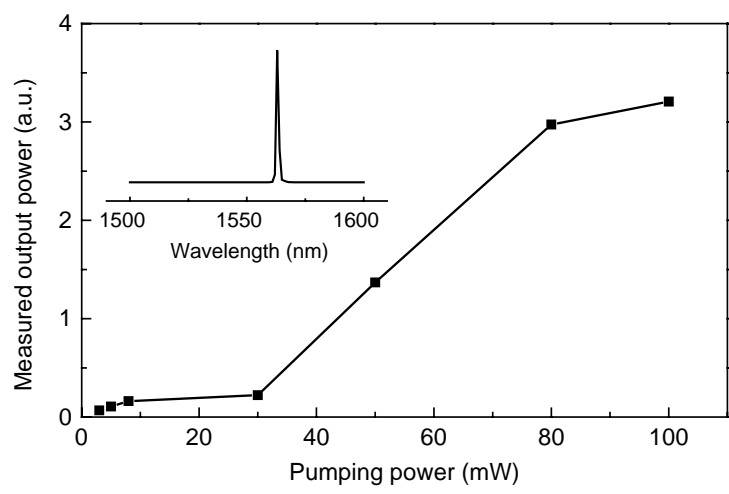

Fig. 8. The VCSEL output power versus input laser pumping power characteristics at room temperature. The inset shows the VCSEL emission spectrum at the pumping power above the threshold. Peak emission wavelength is $1562 \mathrm{~nm}$.

wavelength from the Fabry-Perot dip was attributed to the local heating caused by the strong absorption. The equivalent threshold current density is calculated to be $2 \mathrm{kA} / \mathrm{cm}^{2}$ when taking into account the absorption of the pumping light in the cladding layers and the reflection at the surface. The relatively large threshold current density might be attributed to the non-optimized quantum well structure and the absorption of the pumping power by the $2 \lambda$ thick cladding layers in the laser structure.

\section{Summary}

In summary, we have grown an $\mathrm{InP} / \mathrm{InGaAlAs}$ DBR structure with excellent optical properties using MOCVD and the growth interruption technique. We found that the growth interruption time between InP and InGaAlAs interface was very critical for obtaining high reflectivity. A 35 pairs $1.55 \mu \mathrm{m}$ centered InP/InGaAlAs DBRs grown with the interruption time of $0.4 \mathrm{~min}$ has the stopband of more than $100 \mathrm{~nm}$ and the highest reflectivity exceeding $99 \%$. We also demonstrated 
the optically pumped InP-based VCSELs with the 35 pairs InP/InGaAlAs DBRs and 10 pairs $\mathrm{SiO}_{2} /$ $\mathrm{TiO}_{2}$ top dielectric mirrors and a $2 \lambda$ thick cavity composed of periodic SCMQWs to fully utilize the gain in every quantum well. The optically pumped VCSELs operated at room temperature with the threshold pumping power of $30 \mathrm{~mW}$.

\section{Acknowledgements}

The authors wish to thank the considerable technical support from the Union Optronics Corporation. This work was supported by the National Science Council of Republic of China (ROC) under Contract No. NSC 91-2215-E009030 and by the Academic Excellence Program of the Ministry of Education of ROC under the Contract No. 88-FA06-AB.

\section{References}

[1] D.I. Babic, J. Piprek, K. Streubel, R.P. Mirin, N.M. Margalit, D.E. Mars, J.E. Bowers, E.L. Hu, IEEE J. Quantum Electron. 33 (8) (1997) 1369.

[2] Y. Ohiso, C. Amano, Y. Itoh, H. Takenouchi, T. Kurokawa, IEEE J. Quantum Electron. 34 (10) (1998) 1904.
[3] S. Sato, N. Nishiyama, T. Miyamoto, T. Takahashi, N. Jikutani, M. Arai, A. Matsutani, F. Koyama, K. Iga, Electron. Lett. 36 (24) (2000) 2018.

[4] G. Steinle, F. Mederer, M. Kicherer, R. Michalzik, G. Kristen, A.Y. Egorov, H. Riechert, H.D. Wolf, K.J. Ebeling, Electron. Lett. 37 (10) (2001) 632.

[5] J. Boucart, C. Starck, F. Gaborit, A. Plais, N. Bouche, E. Derouin, J.C. Remy, J. Bonnet-Gamard, L. Goldstein, C. Fortin, D. Carpentier, P. Salet, F. Brillouet, J. Jacquet, IEEE J. Sel. Top. Quantum Electron. 5 (3) (1999) 520.

[6] W. Yuen, G.S. Li, R.F. Nabiev, J. Boucart, P. Kner, R.J. Stone, D. Zhang, M. Beaudoin, T. Zheng, C. He, K. Yu, M. Jansen, D.P. Worland, C.J. Chang-Hasnain, Electron. Lett. 36 (13) (2000) 1121.

[7] E. Hall, H. Kroemer, L.A. Coldren, Electron. Lett. 35 (5) (1999) 425.

[8] E. Hall, S. Nakagawa, G. Almuneau, J.K. Kim, L.A. Coldren, Electron. Lett. 36 (17) (2000) 1465.

[9] I. Sagnes, G. Le Roux, C. Meriadec, A. Mereuta, G. SaintGirons, M. Bensoussan, Electron. Lett. 37 (37) (2001) 500.

[10] M. Linnik, A. Christou, IEEE Trans. Electron Devices 48 (10) (2001) 2228.

[11] M.H.M. Reddy, T. Asano, R. Koda, D.A. Buell, L.A. Coldren, Electron. Lett. 38 (20) (2002) 1181.

[12] K. Streubel, J. Wallin, M. Amiotti, G. Landgren, J. Crystal Growth 124 (1992) 541-546.

[13] K. Streubel, V. Harle, F. Scholz, G. Landgren, J. Appl. Phys. 71 (1992) 3300.

[14] J.H. Baek, I.H. Choi, B. Lee, W.S. Han, H.K. Cho, Appl. Phys. Lett. 75 (11) (1999) 1500.

[15] G. Boehm, M. Ortsiefer, R. Shau, F. Koehler, R. Meyer, M.C. Amann, J. Crystal Growth 319 (2001) 227-228.

[16] J. Piprek, Y.A. Akulova, D.I. Babic, L.A. Coldren, J.E. Bowers, Appl. Phys. Lett. 72 (15) (1998) 1814. 